\title{
Le fratture esposte
}

\author{
Achille Pellegrino \\ U.O.C. di Orto-Traumatologia, Ospedale "S.G. Moscati", Aversa (CE), Italia \\ achpell@tin.it
}

Pubblicato online: 4 marzo 2016

(c) Società Italiana Ortopedici Traumatologi Ospedalieri d'Italia 2016

Fino a 150 anni fa frattura esposta era sinonimo di morte e generalmente comportava l'immediata amputazione dell'arto leso, anche quest'ultima causa di una elevata mortalità, generalmente conseguente a emorragia o infezione: la guarigione di una frattura esposta di femore nel XIX secolo era addirittura considerato un evento eccezionale.

Le conquiste del XX secolo nella cura e profilassi precoce della sepsi con antibiotici, nel perfezionamento delle procedure anestesiologiche e rianimatorie, nella stabilizzazione precoce delle fratture con l'uso di fissatori esterni sempre più maneggevoli e affidabili, nelle tecniche chirurgiche di conversione della fissazione esterna verso sintesi interne più stabili, hanno spostato l'interesse dei traumatologi dal salvataggio della vita umana e dell'arto all'utilizzo di metodiche per ridurre i tempi di consolidazione e di guarigione.

Le fratture esposte sono in costante aumento, perché sono in continua ascesa traumi ad alta energia che avvengono sul lavoro e nel corso di incidenti stradali, così come traumi a media e bassa energia negli anziani per l'allungarsi della vita media.

Il management delle fratture esposte rappresenta, a tutt'oggi, una sfida continua per il chirurgo ortopedico, in un momento così delicato della sanità italiana dove "tagli" e "spending review", "medicina difensiva" e "controversie medico-legali" condizionano sempre più il timing e l'approccio terapeutico fondamentali per evitare temibili complicanze quali la sepsi e la sindrome compartimentale, la pseudoartrosi e la viziosa consolidazione.
In questo numero monotematico, affidato all'A.C.O.T.O., sono state affrontate tutte le problematiche delle fratture esposte a livello dei segmenti scheletrici sia dell'arto superiore che inferiore tenendo conto delle attuali linee guida per un corretto timing, un preciso inquadramento diagnostico, un'adeguata profilassi antibiotica e antitrombotica, un'accurata e tempestiva opzione chirurgica.

Un "GRAZIE" di vero cuore va a tutti i colleghi, di provata capacità ed esperienza, che hanno contribuito alla stesura di questo volume scientifico.

Un ringraziamento particolare va anche all'O.T.O.D.I. che ha accolto la nostra proposta di curare codesto fascicolo monotematico e ai Direttori Scientifici de Lo Scalpello che ci hanno supportato nella elaborazione dei manoscritti.

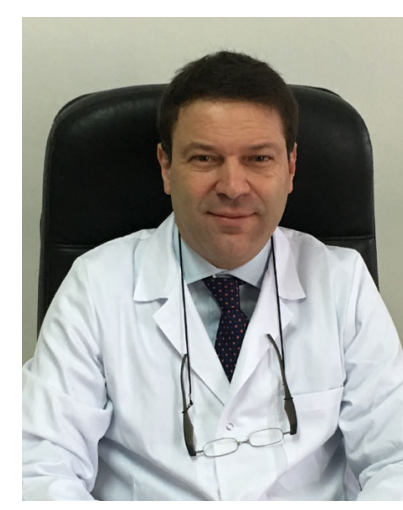

